\title{
Design of Highly Linearity Active Mixer for 4G RF Receiver
}

\author{
Wang Wei \\ College of Electronics Engineering \\ Chongqing International Semiconductor College, \\ Chongqing University of Posts and \\ Telecommunications \\ Chongqing, China \\ wangwei@cqupt.edu.cn
}

\author{
Liang Yao \\ College of Electronics Engineering \\ Chongqing International Semiconductor College, \\ Chongqing University of Posts and \\ Telecommunications \\ Chongqing, China \\ 648140642@qq.com
}

xingchen0932@126.com

Zhao Chen

College of Electronics Engineering

Chongqing International Semiconductor College, Chongqing University of Posts and

Telecommunications

Chongqing, China

\begin{abstract}
A down-conversion active mixer for LTE receiver is proposed, which focus on improving the traditional subthreshold compensation method. The linearity is improved by adjusting the load inductance of transconductance stage and optimizing the phase of third-order transconductance coefficient; the noise is reduced with current injection technology. The designed active mixer is simulated with TSMC 0.18 $\mu \mathrm{m}$ 1P6M CMOS technology. The results show that: the mixer can be operated at the 1.4-3.6 GHz band, and with the IIP3 of 5.6 10.7 dBm, the SSB NF ranges from 9.4 to $13.6 \mathrm{~dB}$, the gain ranges from -0.25 to $3.4 \mathrm{~dB}$, the power consumption is $14.1 \mathrm{~mW}$ with supply of $1.5 \mathrm{~V}$. The proposed mixer also has a merit of low NF and high linearity.
\end{abstract}

Keywords-4G; down-conversion; threshold compensation; current bleeding;linearity

\section{INTRODUCTION}

In recent years, with the growing demands for high quality of communication, the fourth generation of mobile communication $(4 \mathrm{G})$ has been proposed in 2009, which can provide high and fixed data rate with the adoption of MIMO and OFDM. The new communication system can operate at $1.4 \sim 3.6 \mathrm{GHz}$ and supports a variety of bandwidth allocations: $1.4 \mathrm{MHz}, 3 \mathrm{MHz}, 5 \mathrm{MHz}, 10 \mathrm{MHz}, 15 \mathrm{MHz}$ and $20 \mathrm{MHz}$ etc. Compared with $3 \mathrm{G}$, the band width gets broader and data rate gets larger, so the requirements for hardware are increasing high also.

Linearity is an important parameter of the RF receiver, which determines the dynamic range of the input signal ${ }^{[2]}$. Linearity and the isolation between port and port decide the linearity and stability of the receiver. To improve the linearity, many different methods have been proposed in the literatures. The method biasing one of the transistors in strong inversion region and the other one in sub-threshold region in proper workplace to achieve the aim that the third-order transconductance closes to zero is adopted in $[3,4]$. Then the IMD3 gets reduced and the linearity gets improved. However, this method requires a better level of the hardware circuit because of the external factors such as temperature which will cause zero drift. As we know zero drift can lead to floating of the IMD3. Even the design in the [4] just operates at $0.7-2.7 \mathrm{GHz}$; [5] proposed a nonlinear compensation method that gets the IIP3 improved by $4.3 \mathrm{dBm}$ with the using of $L C$ folding load structure. However, the power consumption increases by $10 \%$. [6] proposes a subthreshold MOSFET compensation method, with the principle of making two transistors connect two transistors connected in parallel in different workspaces, in order that both the third-order transconductances can be distributed on both sides of zero. Since the coefficients are vectors, they can be eliminated by adjusting the phase of vectors to reduce IMD3. But the design mainly works for the applications in the $2.4 \mathrm{GHz}$. Also it can not be applied for broadband systems. When the frequency gets higher, the third-order transconductance can not be completely eliminated, leading to a worse performance of the IIP3.

This paper presents a high linearity mixer design which optimizes the subthreshold MOSFET compensation method by substituting tapped inductor for ordinary inductor. Then the phase of the Third-order transconductance will be eliminated with IMD2. The current bleeding technology also is adopted in this design to decrease the flick noise that produced from the common source node of the switch stage.

\section{Circuit ANALYSIS AND Design}

For the active mixer designed for LTE, most of them are based on the active Gilbert mixer structure ${ }^{[7,8]}$. Fig.1 shows the mixer structure used in [6], which is the same with the circuit of the subthreshold MOSFET compensation method. The mainly work is optimizing the linearity of the transconductance stage. The following picture is the analysis of the circuit and the improvement program. 


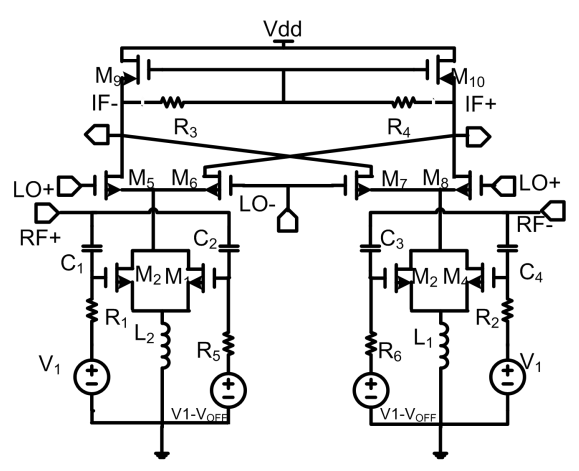

Figure 1. Mixer based on subthreshold MOSFET Compensation

\section{A. Linearity Analysis}

For a MOS transistor operating in the saturation region, the relationship between small signal leakage current and the gate voltage of the signal is given by:

$$
i_{d}\left(v_{g s}\right)=g_{1} v_{g s}+g_{2} v_{g s}^{2}+g_{3} v_{g s}^{3}+\ldots
$$

$g_{1}$ is the transconductance and $g_{2}, g_{3}$ are the higher order coefficients. Among them, $g_{3}$ has the greatest impact on the IIP3 . Without the case of the high frequency signal, the IIP3 can be simply expressed as:

$$
A_{I P 3}=\sqrt{\frac{4}{3} \cdot\left|\frac{g_{1}}{g_{3}}\right|}
$$

$g_{3}$ mainly dependents on the gate-source $\left(V_{\mathrm{gs}}\right)$ and drain-source $\left(V_{\mathrm{ds}}\right)$ voltage. However, when the MOS transistor operates in the saturation region, the impact of drain-source voltage can be ignored, and then the each order of the transconductances coefficients can be expressed as:

$g_{1}=\frac{\partial I_{D}}{\partial V_{G S}} \quad g_{2}=\frac{1}{2} \cdot \frac{\partial^{2} I_{D}}{\partial V_{G S}^{2}} \quad g_{3}=\frac{1}{6} \cdot \frac{\partial^{3} I_{D}}{\partial V_{G S}^{3}}$

When the transistor trans from weak inversion region or moderate inversion region to strong inversion region while under the action of $V_{\mathrm{GS}}, g_{3}$ also changes from positive to negative.

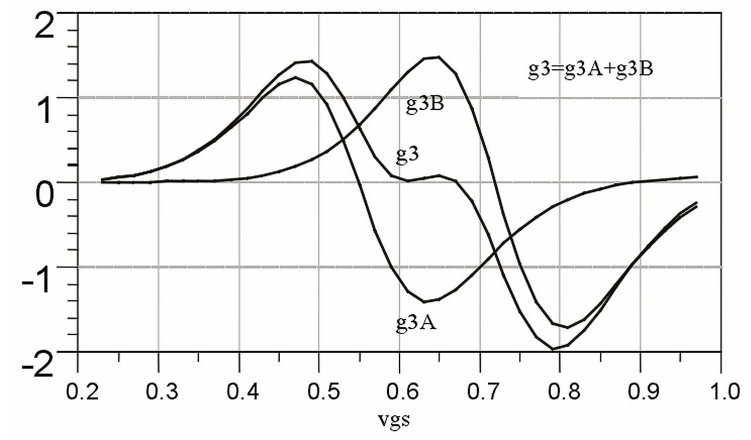

Figure 2. Third-order transconductance versus the bias voltage

As shown in the Fig .2, if the $g_{3}$ of one transistor is biased in the positive region, the other one is biased in the negative region. Then both of them can offset each other to get a combination of them, which is close to zero. Ideally, $g_{3}$ tends to be zero and $A_{\mathrm{IP} 3}$ can achieve an infinite value. Fig. 3 shows the circuit arrangement:

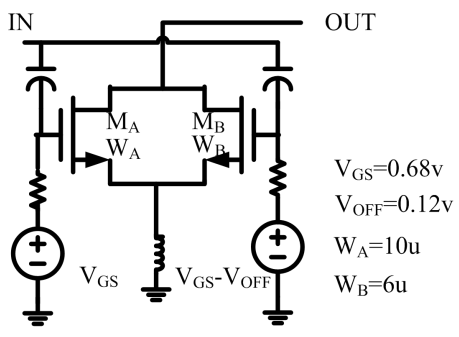

Figure 3. Subthreshold MOSFET compensated transconductance stage

$M_{\mathrm{A}}, M_{\mathrm{B}}$ are biased in different regions, making sure that the transconductance of $M_{1}, M_{2}$ are located in strong inversion and weak inversion region respectively. So we can get, then $g_{3 \mathrm{~A}}$ and $g_{3 \mathrm{~B}}$ will mutually offset. The total output third-order transconductance can be expressed as:

$$
g_{3}=g_{3 A}+g_{3 B}
$$

When $V_{\text {gs }}$ is $0.63 \mathrm{~V}, \mathrm{~g} 3$ tends to be zero. Then we can get a larger $A_{\mathrm{IP} 3}$. When considering the case of high frequency, Fig. 4 shows the small-signal model:

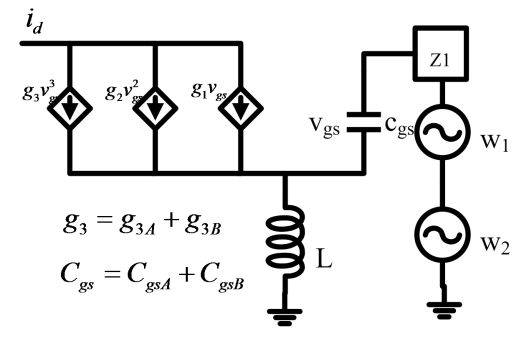

Figure 4. nonlinear small-signal model

In the small-signal nonlinear model, we neglect the impact of $C_{\text {gd. }}$. All the IMD3 is produced in $g_{3} v^{3}{ }_{g s}$ when there is no $L$. The source inductor $L$ forming a negative feedback circuit and the feedback gets more obvious impact on the high frequency signal. For example: the second harmonic generated by $g_{2} v_{g s}^{2}$ will produce thirdorder intermediation signals, so the second harmonic can also generate nonlinear IMD3.

Since $\triangle \omega \quad\left(=\omega_{2}-\omega_{1}\right)$ is much smaller than $\omega_{2}$ and $\omega_{1}$, then we can get $j \Delta w L \approx 0$. When the circuit is matched to the signal source, we can obtain IIP3 as follows:

$$
\begin{gathered}
I I P_{3}=\frac{4 g_{1}^{2} \omega^{2} L C_{g s}}{3|\varepsilon|} \\
\varepsilon=g_{3}-\frac{2 g_{2}^{2} / 3}{g_{1}+\frac{1}{j 2 \omega L}+j 2 \omega C_{g s}+Z_{1}(2 \omega) \frac{C_{g s}}{L}}
\end{gathered}
$$

From (5) and (6), we can see that where $L$ is the source inductor the $\varepsilon$ cannot be zero even by setting the bias voltage only. In (6), the latter half is not only determined by the third-order transconductance. However, from the foregoing, the second harmonic generates again after mixing. In this regard, it is not effective to improve 
IIP3 by relying on the DC circuit only.

By reducing the scale of the source inductor, we can reach the aim of improving the IIP3. However, the value of the $Z_{1}(2 \omega)$ would get larger too. What's worse, the feedback of $C_{\mathrm{gd}}$ gets enhanced which leads to another frequency mixing which would contribute to $I M D 3$. So simple to reduce value of the inductance is difficult to enhance IIP3. In [6], although the method has improved the linearity, as the inductance value is so small, the performance of the $N F$ and the VSWR is not very satisfactory.

\section{B. Noise Analysis}

For MOS, the main sources of noise are the induced gate noise and the noise generated from the leakage current in the RF circuits:

$$
\begin{aligned}
& \overline{i_{n d}^{2}}=4 k T \Delta f \gamma g_{d 0} \\
& \overline{i_{n g}^{2}}=\frac{4}{5} \frac{k^{2} T^{2} \Delta f \delta \gamma \omega^{2} C_{g s}^{2}}{I_{D . s a t} \cdot q}
\end{aligned}
$$

$\gamma$ and $\delta$ are independent bias noise factor, and $g_{\mathrm{d} 0}$ is the transconductance when $V_{\text {ds }}$ equals to zero. When $M O S$ is biased in strong inversion region:

$$
g_{d 0}=\frac{2 I_{D . s a t}}{V_{G S}-V_{T H}}
$$

Where $I_{\mathrm{D} \text {.sat }}$ is the drain current, $V_{\mathrm{TH}}$ is the threshold voltage. The two noise currents in equations (7) and (8) are partially correlated, and the correlation coefficient is:

$$
c=\frac{i_{n g} \cdot i_{n d}^{*}}{\sqrt{i_{n g}^{*} \cdot i_{n d}^{*}}}
$$

For long channel MOS transistor biased in saturated region, the $\gamma$ and $\delta$ are $2 / 3,4 / 3$. For short channel transistors, $\gamma$ and $\delta$ can be achieved 2-3 times than those. For simplicity, the short channel effect is ignored here. The $\gamma$ and $\delta$ are derived as $1 / 2,5 / 4$. Since current of the transistor biasing in weak inversion is diffusion current, $\mathrm{g}_{\mathrm{d} 0}$ takes $\mathrm{I}_{\text {D.sat }} / \Phi_{\mathrm{t}}$, where $\Phi_{\mathrm{t}}$ is the thermal voltage coefficient and equals to $\mathrm{kT} / \mathrm{q}$ :

$$
g_{d 0}=\frac{I_{D . s a t}}{\phi_{t}}
$$

Take the (11) into (8), we can obtain:

$$
\begin{aligned}
\overline{i_{n g}^{2}} & =4 k T \Delta f \delta \gamma \frac{\omega^{2} C_{g s}^{2}}{\frac{5 I_{D . s a t} \cdot q}{K T}} \\
& =\frac{4}{5} \frac{k^{2} T^{2} \Delta f \delta \gamma \omega^{2} C_{g s}^{2}}{I_{D . s a t} \cdot q}
\end{aligned}
$$

What can be seen from the above equations, the induced gate noise in weak inversion region of the $M O S$ transistor will decrease with the increasing of the diffusion current. Flicker noise in the strong inversion region of the transistor is ${ }^{[9]}$ :

$V_{1 / f_{-} \text {out }}=\frac{4 I_{L O}}{S \cdot T_{L O}} \frac{2 K_{N}}{C_{O X}(W L)_{S W}} \frac{1}{f}+\frac{2 C_{P}}{T_{L O}} \frac{2 K_{N}}{C_{O X}(W L)_{S W}} \frac{1}{f}$

The $S \cdot T_{L O}$ can be seen as $4 A$, where $A$ is the amplitude of the oscillation. When the transistor is in strong inversion region, the flicker noise and leakage current fall in a negative correlation. In the circuit designed of this paper, as the use of current injection technology, the current source Shares the current by $60 \%$, reducing the impact of the flicker noise:

\section{MIXER CIRCUIT DESIGN}

The mixer circuit structure that has been improved is shown in Fig. 5:

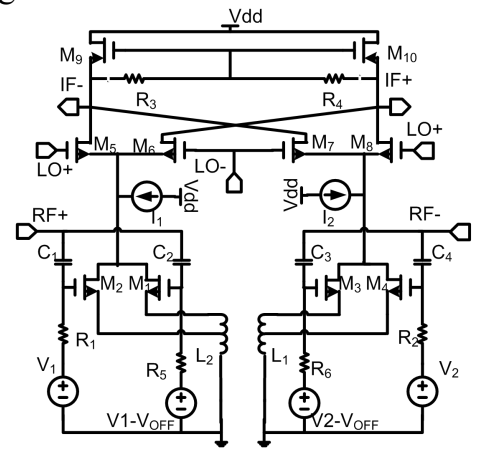

Figure 5. the proposed improved mixer circuit

In this circuit configuration, $M_{9} \sim M_{10}$ are the loads; $M_{5} \sim M_{8}$ are switch stage transistors, which are the input terminal of oscillation signal; $R_{1} \sim R_{6}$ are protection resistors; $V_{1}, V_{1}-V_{\text {OFF }}$ are the bias voltages; $C_{1} \sim C_{4}$ are blocking capacitors; $M_{1} \sim M_{3}$ are biased in the saturation region; $M_{2} \sim M_{3}$ are biased in the subthreshold region; $L_{1} \sim L_{2}$ are tapped inductor. By adjusting the access inductance values of $M_{2} \sim M_{3}$, the phase deflection of third-order transconductance can be achieved.

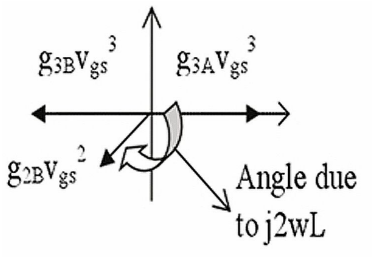

a

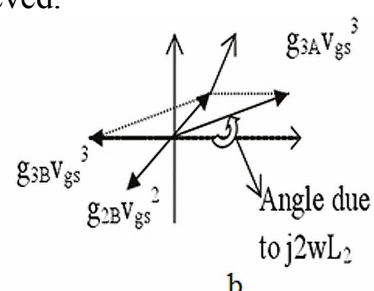

Figure 6. the phase deflection of third-order transconductance

Fig. 6(a) shows the second and third transconductance phase diagram. Fig. 6(b) shows those of the circuit proposed in this paper. Compared with chart (a), by adjusting the access value of the tapped inductor, we can change phase direction of the third-order transconductance. Then the phase of the third transconductance of $M_{1}$ and $M_{2}$ can be rotated to be eliminated. The linearity gets improved. $I_{1} \sim I_{2}$ are current sources.

\section{MEASUREMENT RESULTS}

The designed MIXER was manufactured in a $0.18 \mu \mathrm{m}$ CMOS technology. In order to evaluate the mixer performance, external matching circuit was designed. As the tapped inductor was adopted, the value of the IMD3 was drastically reduced, leading to the greatly enhance of $I I P 3$. As shown in the fig. 7, the IIP3 increases by $4 \mathrm{dBm}$, reaching to $10.7 \mathrm{dBm}$ with the adoption of the tapped inductor. At the same time, the current injection structure shares the current by $2.8 \mathrm{~mA}$, so that the current of the switch stage can be greatly reduced, which could optimize the performance of the $N F$. Fig. 8 shows the $S S B N F$, from 
which we can see that the $N F$ gets decreased by $5.5 \mathrm{~dB}$ and becomes more flat. Although the power consumption increased by $28 \%$, but relative to the improvements of the performance, the sacrifice is still quite valuable. In the frequency range from 1.4 to $3.6 \mathrm{GHz}$, the consumption is $14.1 \mathrm{~mW}$, while with a supply voltage of $1.5 \mathrm{~V}$. Fig. 9 shows that the $R F$ and $L O$ input reflection coefficient are located within the scope less than-10dB. Fig. 10 shows that the conversions gain curve with $L O$ power, from which we can see that the peak position of the gain varies with the operating frequency. In order to balance the performance of the parameters, here we take the oscillator power at $7 \mathrm{dBm}$. From the figure we can see that the conversion gain range from -0.25 to $2.4 d B$. TABLE I compares the performances between the proposed mixer and the mixer in the literature.

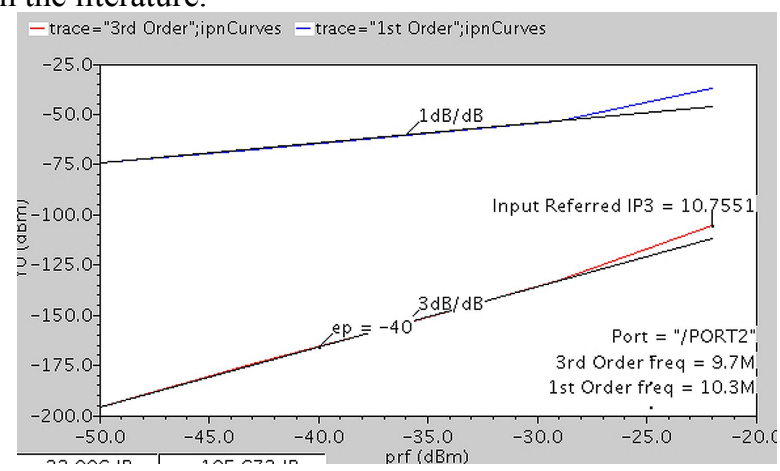

Figure 7. (a) IIP3 improved

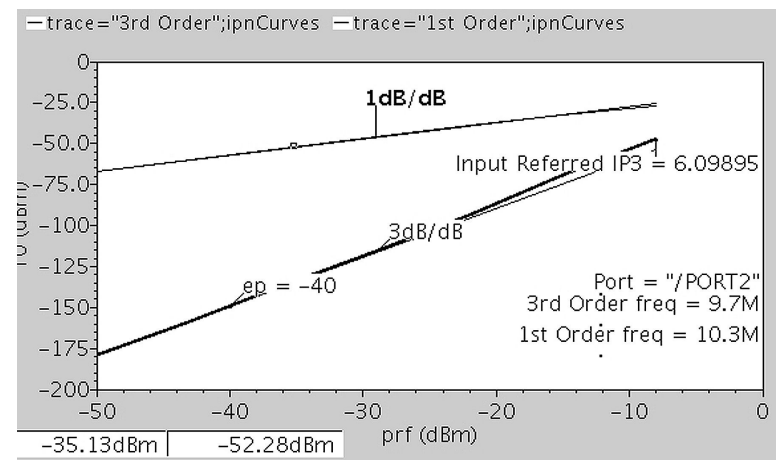

Figure 7. (b) IIP3 before improved

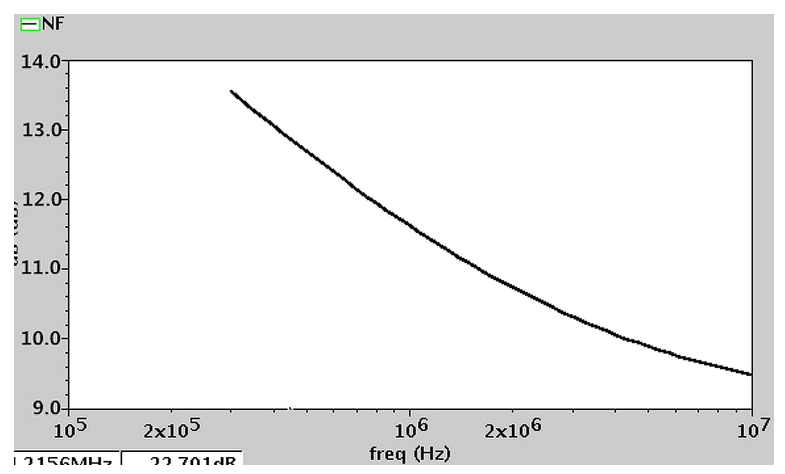

Figure 8. (a) SSB NF improved
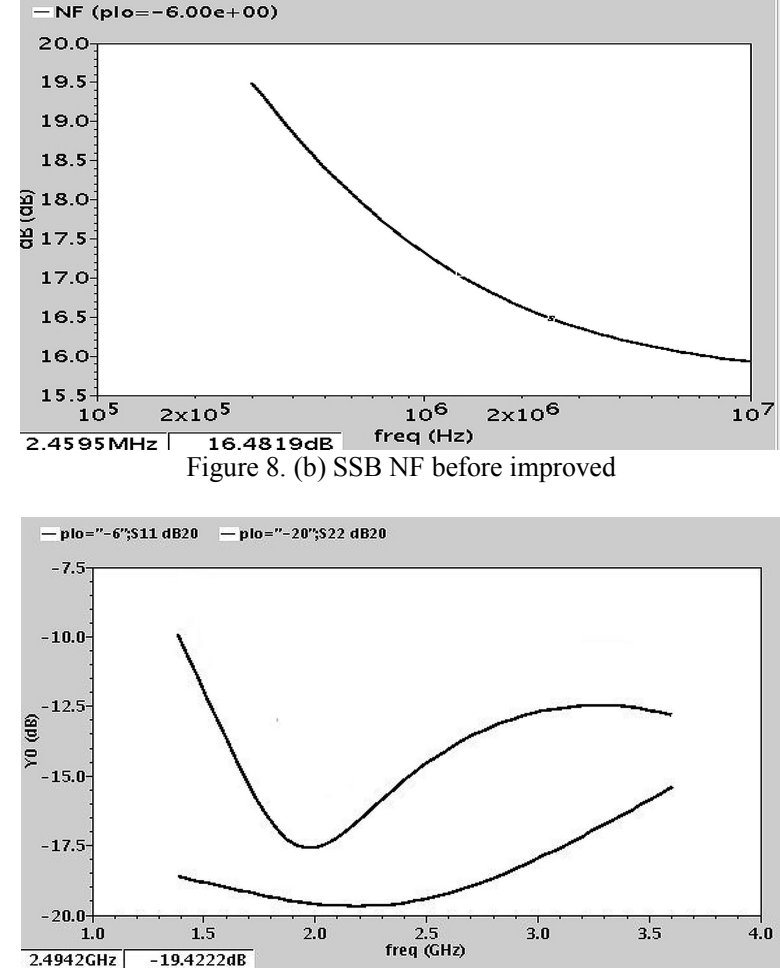

Figure 9. (a) RF S11, Lo S22

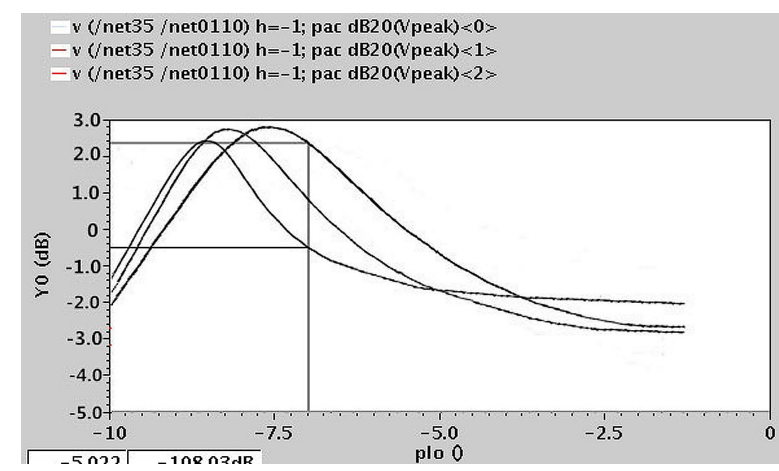

Figure 10. Conversion gain versus LO power at different frequencies

TABLE I. PERFORMANCE COMPARISON OF DIFFERENT MIXER PUBLISHED IN RECENT YEARS AND THIS WORK

\begin{tabular}{|c|c|c|c|}
\hline & ref[10] & ref[11] & this \\
\hline Tech $(u m)$ & 0.13 & 0.18 & 0.18 \\
\hline $\mathrm{Vol}(\mathrm{V})$ & 1.2 & 1.2 & 1.5 \\
\hline $\mathrm{f}_{\mathrm{RF}}(\mathrm{GHz})$ & $1.4 \sim 3.6$ & $1.4 \sim 3.6$ & $1.4 \sim 3.6$ \\
\hline $\mathrm{f}_{\mathrm{LO}}(\mathrm{GHz})$ & $1.39 \sim 3.59$ & $1.39 \sim 3.59$ & $1.39 \sim 3.59$ \\
\hline $\mathrm{P}_{\mathrm{LO}}(d B m)$ & $\mathrm{N} / \mathrm{A}$ & -8 & -7 \\
\hline $\mathrm{IIP} 3(d B m)$ & $-13 \sim-10$ & $-0.5 \sim 2.6$ & $5.6 \sim 10.7$ \\
\hline $\mathrm{P}_{-} d B(d B m)$ & $\mathrm{N} / \mathrm{A}$ & $-12.9 \sim 13.9$ & $-11.53 \sim 2.3$ \\
\hline $\mathrm{Gain}(d B)$ & $6.5 \sim 7$ & $4.3 \sim 6.9$ & $-0.25 \sim 2.4$ \\
\hline $\mathrm{NF}_{\mathrm{SSB}}(d B m)$ & $2.7 \sim 6.5$ & $20.8 \sim 23.2$ & $9.4 \sim 13.6$ \\
\hline
\end{tabular}

\section{CONCLUSION}

In this paper, subthreshold MOSFET compensation method was analyzed and improved: By replacing the ordinary inductor into tapped inductor to optimize the feedback of the transconductance stage, which purpose is to adjust the phase direction of the transconductance, so that the sum of the transconductance coefficients closes to 
zero to improve the linearity. The use of the current injection technology effectively reduces the noise factor. The stimulation results show that: the linearity of the proposed mixer gets improved by $4 \mathrm{dBm}$; the singlesideband noise figure decreases by $5.5 \mathrm{~dB}$, which is suit for the application of the LTE.

\section{REFERENCES}

[1] S. Sesia, I. Toufik, and M. Baker, LTE: the UMTS long term evolution: From Theory to Practice [M]. New York: John Wiley \& Sons, 2009. 7-20.

[2] E.S. Atalla, A. Bellaouar, P.T. Balsara, IIP2 requirements in 4G LTE handset receivers[C], IEEE 56th Int. Midwest Symposium on Circuits and Systems, 2013, 1132-1135.

[3] V. Aparin, G. Brown, L.E. Larson, Linearization of CMOS LAN's via optimum gate biasing[C], IEEE Proceedings of the 2004 International Symposium on Circuits and Systems, 2004, 4: 748751.

[4] H.N. Nguyen, V.H. Le, K.U. Gwak, et al. Low power, high linearity wideband receiver front-end for LTE application[C],13th International Conference on Advanced Communication Technology, 2011, 640-643.
[5] Cao Ke, Yang Huazhong, Wang Hui. Design of Low-Voltage Low Noise Amplifiers with High Linearity[J].Chinese journal of semiconductors, 2004, 25(11): 1364-1369。

[6] T.W. Kim, B. Kim, K. Lee. highly linear receiver front-end adopting MOSFET transconductance linearization by multiple gated transistors. IEEE J. Solid-State Circuits, 2004, 39(1): 223229.

[7] H.C. Wei, R.M. Weng, C.L. Hsiao, et al, A 1.5 V $2.4 \mathrm{GHz}$ CMOS mixer with high linearity[C], The 2004 IEEE Asia-Pacific Conference on Circuits and Systems, 2004, 1:289-292.

[8] H.C. Wei, C.L. Hsiao, R.M. Weng. A broadband high linearity current-reuse bulk-controlled mixer for 4G applications[J]. Progress In Electromagnetics Research, 2013, 138.337-350.

[9] V. Vidojkovic, V.D. J. Tang, A. Leeuwenburgh, et al, A lowvoltage folded-switching mixer in $0.18-\mu \mathrm{m}$ CMOS[J]. IEEE J. Solid-State Circuits, 2005, 40(6): 1259-1264.

[10] D.H. Na, T.W. Kim. A 1.2 V, 0.87-3.7 GHz wideband low-noise mixer using a current mirror for multiband application[J]. IEEE Microwave and Wireless Components Letters, 2012, 22(2): 91-93.

[11] H.C. Wei. A $1.2 \mathrm{~V}$ High Linearity Down-Conversion Mixer for LTE-advanced Applications[J]. 2011, 19:102-106. 